\title{
Leader
}

\section{Nasal manifestations of rheumatic diseases}

What should a rheumatologist know about nasal disorders? In a significant minority of patients with rheumatological disorders nasal symptoms and signs are part of the picture and can help in establishing a diagnosis.

To illustrate this point, over $50 \%$ of patients with Wegener's granulomatosis (WG) have nasal symptoms and signs at presentation and these have a positive predictive value of $63 \%$, while a positive nasal biopsy has a positive predictive factor of $100 \% .^{1}$ The most common nasal symptoms in these patients are nasal obstruction and discharge but it is the unusual symptom of crusting that should alert the clinician and helps differentiate these patients from those with other more common rhinological conditions. (This is important as approximately $19 \%$ of most populations have nasal symptoms and nasal obstruction as part of their rhinosinusitis. ${ }^{2}$ The majority have seasonal allergic rhinitis $(16 \%)$ while the remainder have perennial allergic rhinitis, chronic infective rhinosinusitis, idiopathic rhinitis or systemic disease that affects the nose. ${ }^{3}$ ) Apart from nasal crusting or progressive bilateral nasal obstruction, other symptoms of WG include a minor epistaxis, whistling if there is a septal perforation, a reduction in sense of smell, and through this taste.

You do not need any sophisticated equipment to inspect the nasal mucosa. An otoscope with a large speculum allows a good view of the nasal mucosa as long as the patient is asked to breathe through their mouth to avoid condensation on the lens. Alternatively elevate the tip of the nose upwards with your thumb and inspect the anterior third of the nose with the light from a pen torch. The mucosa in WG has a granular quality and stagnant mucus superinfected with nasal commensals from the nasal vestibule can coat the septum and lateral nasal wall (see fig 1). A septal perforation is a stronger sign, as is supratip depression (collapse of the dorsal profile as a result of loss

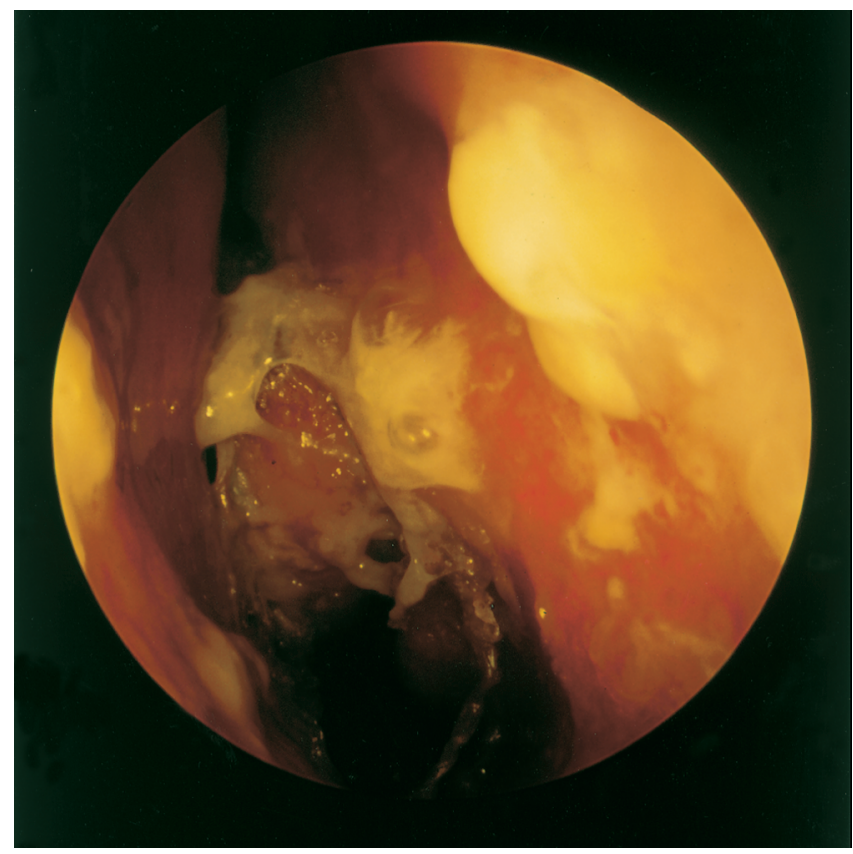

Figure 1 Crusting and mucus stagnation with erythematous granular mucosa in the left nasal airway. Wegener's granulomatosis. of cartilagenous support), particularly if there is no history of nasal trauma or septal surgery. Nasal adhesions from the lateral nasal wall to the septum occur spontaneously. However, it should be noted that an absence of nasal symptoms and signs should be treated with caution as in a recently reported series of 53 patients with WG the negative predictive value was only $41 \%$, a negative c-ANCA $79 \%$, and a negative nasal biopsy $74 \%$. While a positive c-ANCA is highly indicative of WG, approximately $10 \%$ take up to four years to become positive. ${ }^{1}$ Other ENT manifestations are subglottic stenosis, hearing loss either because of otitis media with effusion or a sensorineural hearing loss, the latter requiring systemic treatment. Nasal surgery for adhesions or reconstruction should be deferred until the disease has been quiescent for years rather than months otherwise severe intranasal adhesions form and if a graft is used it resorbs.

A nasal biopsy specimen should be obtained in any patient where there is a suspicion of a vasculitis where there are nasal signs and doubt remains about the diagnosis. When there are signs of granular nasal mucosa, a biopsy is both a specific and sensitive way of diagnosing WG but where there are no symptoms or signs then a biopsy is unlikely to help. The site is readily inspected and biopsied under local anaesthesia. The biopsy specimen should include adjacent normal tissue and not just a "punch" biopsy specimen from the ulcerated edge of a perforation.

The other relevant nasal conditions that coexist with rheumatological disorders and occur, in order of frequency, include sarcoidosis, overlap syndromes, ChurgStrauss syndrome, relapsing polychondritis and systemic lupus erythematosus. ${ }^{4}$

In sarcoidosis nasal symptoms and signs occur in $1-6 \%$ of affected patients ${ }^{5}$ and these in themselves may be indistinguishable from WG (crusting, epistaxis and bilateral

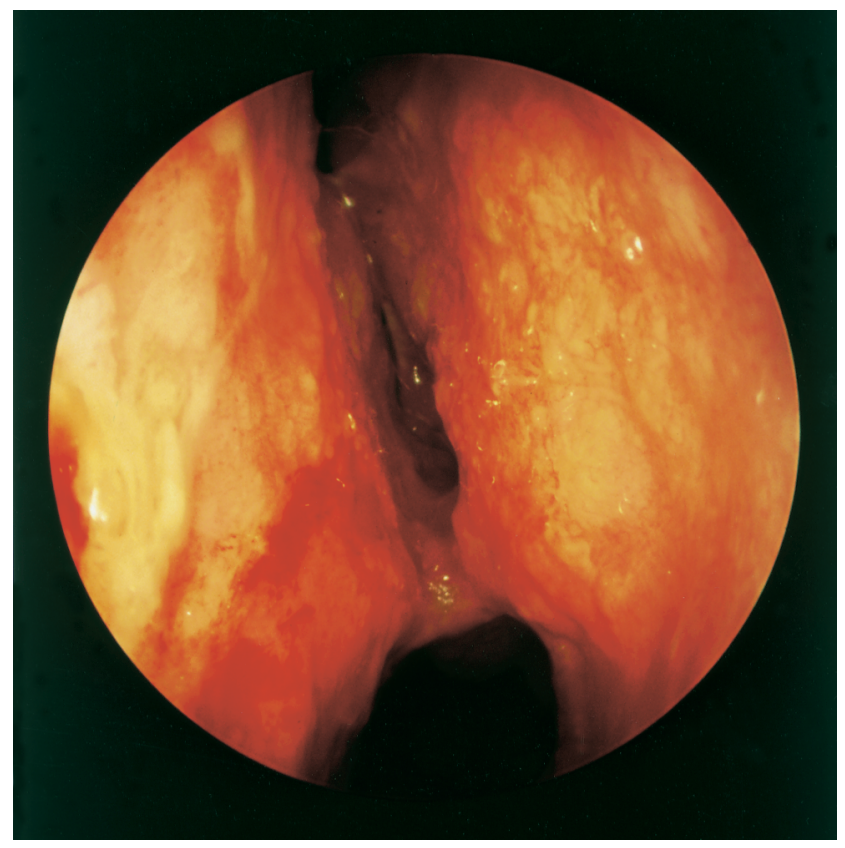

Figure 2 Granular mucosa with tiny yellow submucosal nodules in the left nasal airway. Sarcoidosis. 
obstruction) although distinctive tiny yellow submucosal nodules can sometimes be seen (see fig 2). The overall clinical picture is often different as these patients usually look and feel well and a septal perforation or nasal collapse is less common than in WG. Patients with nasal sarcoid tend to follow an indolent benign course, they rarely have erthema nodosum, and are older (mean age 40) than the majority of patients with sarcoid. The diagnosis depends on the history and the presence of non-caseating granulomas on biopsy and other granulomatous diseases must be excluded. Chest radiographs show changes in approximately $80 \%$ and the angiotensin converting enzyme is often increased. Random nasal biopsy gives a positive diagnosis in $30-58 \%{ }^{6}$ while those with abnormal mucosa give a high diagnostic yield. ${ }^{7}$ Having sought respiratory and other systemic involvement such as hypercalcaemia, and appropriately treated these if necessary, nasal sarcoidosis is best managed with topical nasal corticosteroids although occasionally low doses of oral corticosteroids are needed for symptomatic control. Other ENT manifestations occur in $10-15 \%$ of patients with sarcoidosis and include salivary gland enlargement, cervical lymphadenopathy, lacrimal gland enlargement, facial nerve palsy and lupus pernio (chronic violaceous skin changes from small nodules to large plaques). ${ }^{8}$

Overlap syndromes have a variety of features consistent with different aspects of connective tissue diseases but they are disparate enough to prevent categorisation and sporadically nasal symptoms occur or a septal perforation is found. A nasal biopsy is usually warranted to exclude WG but is rarely of any other benefit. Repair of a septal perforation in these patients is doomed to failure and they are persistently troubled by nasal crusting and bleeding. Nasal douches (half a teaspoon of salt as well as sugar and sodium bicarbonate in 1 litre of boiled water left to cool or proprietary saline sprays are now available) help clear dried and stagnant mucus and moisturising agents applied afterwards such as vaseline sniffed up also helps reduce these symptoms.

Churg-Strauss syndrome is rare and typically the patient has marked nasal polyposis and asthma with eosinophilia but they are systemically unwell and may have a pyrexia of unknown origin. A nasal biopsy is usually helpful in establishing a diagnosis.

Textbooks describe relapsing polychondritis as having chondritis and collapse of their pinna, nose, larynx and costal cartilages. However, this complete clinical picture is rare. Usually the pinna is initially affected on its own and frequently an incorrect diagnosis of infective perichondritis is made as initially they can look the same and infection is far more common. It often takes weeks of failing to respond to antibiotics and the cartilage to start to crumple before the diagnosis is considered and a biopsy arranged. All too often the histological picture is not diagnostic. It can occur along with other connective tissue disorders. For the diagnosis to be made at least three of the following have to be present: chondritis of the pinna, the nose, the larynx or trachea, ocular involvement, a seronegative arthritis or a sensorineural hearing loss with or without vertigo. ${ }^{9}$

In systemic lupus erythematosus the skin of the nose and nasal vestibule can be involved but this is usually long after the diagnosis has become apparent through its other manifestations. In Behçet's syndrome septal ulceration can occur but this is often overshadowed by the presence of severe orogenital ulceration.

In summary, it is worth asking patients with suspected connective tissue disorders if they have any nasal symptoms. If they do, either look for signs of nasal disease yourself, or ask an ENT colleague for an opinion. Interdisciplinary collaboration between clinicians with an interest in this area benefits patients. If there is evidence of abnormal mucosa, a biopsy may help in arriving at a diagnosis. Although histological examination is not a very sensitive test, a positive result has a very high predictive value. A negative biopsy, even from macroscopically abnormal mucosa, does not exclude a vasculitis. Patients with suspected WG and a negative ANCA and a negative biopsy should have their ANCA repeated as it can become positive up to four years after presentation.

N S JONES Department of Otorhinolaryngology, Queen's Medical Centre, Nottingham Correspondence to: Department of Otolaryngology/Head and Neck Surgery, Queen's Medical Centre, Nottingham NG7 2UH.

1 Jennings CR, Jones NS, Dugar J, Powell RJ, Lowe J. Wegener's granulomatosis-a review of diagnosis and treatment in 53 subjects. Rhinology 1998;36:188-91.

2 Jones NS, Carney AS, Davis A. The prevalence of allergic rhinosinusitis: a review. J Laryngol Otol 1998;112:1019-30.

3 Jones NS, Smith PA, Carney SA, Davis A. The prevalence of allergic rhinitis and symptoms in Nottingham. Clin Otolaryngol 1998;23:547-54.

4 Jones NS. Systemic disease and nasal pathology. In: Phillips DE, Jones AS, Hilgers FJM, eds. Diseases of the head and neck, nose and throat.. London Edward Arnold, 1997:884-98.

5 McCaffrey TV, Donald TJ. Sarcoidosis of the nose and paranasal sinuses. Laryngoscope 1983;93:1281-4.

6 Lazarus A. Sarcoidosis. Otolaryngol Clin North Am 1982;15:621-33.

7 Wilson R, Lund V, Sweatman M, Mackay IS, Mitchell DN. Upper respiratory involvement in sarcoidosis and its management. Eur Respir J 1988;1: 69-72

8 Shah UK, White JA, Gooey JE, Hybels RL. Otolaryngologic manifestations of sarcoidosis: presentation and diagnosis. Laryngoscope 1997;107:67-75 9 McAdam LP, O'Hanion MA, Bluestone R, Pearso CM. Relapsing polychondritis: prospective study of 23 patients and a review of the literature. Medicine 1976;55:193-215. 